\title{
Review: longer bed rest does not prevent more postpuncture headaches than immediate mobilisation or short bed rest
}

\author{
Thoennissen J, Herkner H, Lang W, et al. Does bed rest after cervical or lumbar puncture prevent headache? A systematic \\ review and meta-analysis. CMAJ 2001 Nov 13;165:1311-6.
}

\section{QUESTION: In patients having cervical or lumbar puncture, is longer bed rest more effective than immediate mobilisation or short bed rest in preventing headache?}

\section{Data sources}

Studies were identified by searching Medline (1966 to May 2001), EMBASE/Excerpta Medica (1988 to March 2001), Pascal Biomed (1996 to February 2001), Current Contents (1997 to September 1999), PsycINFO (1966 to May 2001), the Cochrane Controlled Trial Register (last search May 15, 2001) and bibliographies of relevant textbooks and retrieved studies.

\section{Study selection}

Studies were selected if they were randomised controlled trials (RCTs), enrolled patients having cervical or lumbar puncture for any reason, compared longer bed rest with immediate mobilisation or short bed rest, and reported occurrence of headache in absolute numbers.

\section{Data extraction}

Data were extracted on patient characteristics, reason for puncture, study quality, interventions, and occurrence of headache by 2 independent reviewers using a standard form.

\section{Main results}

16 RCTs met the selection criteria; 1128 patients were assigned to long bed rest and 1083 patients were assigned to immediate mobilisation or short bed rest. Cervical or lumbar puncture was used for anaesthesia (5 trials), myelography (6 trials), and diagnostic reasons (5 trials). 11 trials compared bed rest with immediate mobilisation (0.5-24 h), and 5 trials compared longer bed rest (4-24 h) with short bed rest (from $0.5-8 \mathrm{~h}$ ). In the 5 trials where reason for puncture was anaesthesia, data were not pooled because clinical heterogeneity existed; none of the trials showed superiority of longer bed rest ( $24 \mathrm{~h}$ in all 5 trials) over immediate mobilisation or short bed rest (up to $8 \mathrm{~h}$ ) in preventing postpuncture headache. In the 6 trials where reason for puncture was myelography, longer bed rest $(12 \mathrm{~h}$ in 1 trial and $24 \mathrm{~h}$ in 5 trials) was not more effective in preventing postpuncture headache than immediate mobilisation (table). In the 5 trials where puncture was done for diagnostic reasons, longer bed rest (30 min in 1 trial and $4-24 \mathrm{~h}$ in 4 trials) was not more effective in preventing postpuncture headache than short bed rest (immediate mobilisation in 4 trials and $30 \mathrm{~min}$ in 1 trial) (table).

\section{Conclusion}

No evidence exists to show that longer bed rest is more effective than immediate mobilisation or short bed rest in preventing headache after cervical or lumbar puncture.
Longer bed rest v immediate mobilisation or short bed rest for preventing headache after cervical or lumbar puncture*

\begin{tabular}{ll} 
Reason for puncture & Pooled relative risk $(95 \% \mathrm{Cl})$ \\
\hline Myelography & $0.93(0.81$ to 1.08$) \dagger$ \\
\hline Diagnosis & $0.97(0.79$ to 1.19$) \dagger$ \\
\hline
\end{tabular}

${ }^{*}$ Abbreviation defined in glossary.

tNot significant

\section{COMMENTARY} findings. ture for anaesthetic reasons. careful evaluation. Lancet 1999;354:1229-33. American Academy of Neurology. Neurology 2000;55:909-14. thesiol Reanim 1991;16:375-8.
Source of funding: no external funding.

For correspondence: Dr M Müllner, University Klinik für Notfallmedizin, Vienna Austria. marcus.muellner@univie.ac.at

A modified version of this abstract appears in ACP Journal Club.

Thoennissen et al provide important information for the management of patients having cervical or lumbar puncture, and add to the evidence from 2 previous reviews. ${ }^{12}$ The authors have sensibly grouped trials by the purpose of the procedure (anaesthesia, myelography, or diagnosis). The clinical heterogeneity was greatest in the group having the procedure for anaesthesia, with 3 different durations of shorter bed rest. Thus, no overall estimate of the effect of bed rest was made for these patients. Bed rest does not seem to reduce headache in patients having lumbar or cervical puncture for myelographic or diagnostic reasons. With the exception of 1 trial, patients in the intervention group were mobilised immediately. These results may be weakened by inadequate allocation concealment or inadequate blinding in the included studies, although the results of the individual trials are remarkably consistent. In no trial did bed rest reduce headaches. Analysis of the potential for publication bias showed that if publication bias were present, it was unlikely to influence the

Clinically, duration of bed rest after cervical or lumbar puncture is an area of considerable practice variation. Use of short term bed rest or immediate mobilisation after cervical or lumbar puncture may reduce the incidence of adverse events associated with prolonged bed rest and the resulting costs to service. For patients, the discomfort of prolonged bed rest could also be prevented. Some evidence exists to suggest that prolonged bed rest may even cause headache in some patient groups. ${ }^{3}{ }^{4}$ Clinical guidelines for patients having cervical or lumbar puncture should be reviewed and adjusted to recommend immediate mobilisation or short bed rest after the procedure for patients having myelography or diagnostic punctures. Further research is required before a similar recommendation could be made for patients having a punc-

John McArthur, RN, MHSc(Hons) Clinical Services Manager Dunedin Hospital, New Zealand

1 Allen C, Glasziou P, Del Mar C. Bed rest: a potentially harmful treatment needing more

2 Evans RW, Armon C, Frohman EM, et al. Assessment: prevention of post-lumbar puncture headaches: report of the Therapeutics and Technology Assessment Subcommittee of the

3 Fassoulaki A, Sarantopoulos C, Andreopoulou K. Is early mobilization associated with lower incidence of postspinal headache? A controlled trial in 69 urologic patients. Anaes-

4 Jensen TT, Eggert W, Hansen KF, et al. [Side-effects of lumbar metrizamide myelography in ambulant patients and patients confined to bed]. Ugeskr Laeger 1987;9:2016-7. 